\title{
Explicit solutions and conservation laws of the logarithmic-KP equation
}

\author{
Yong Wang ${ }^{*}$ (D) and Jiahao Deng
}

"Correspondence:

wangyongbit@163.com

School of Mechatronic Engineering,

Beijing Institute of Technology,

Beijing, 100081, P.R. China

\begin{abstract}
In this paper, we study the logarithmic-KP equation. The analysis depends mainly on the Lie symmetry method. The corresponding vector fields and symmetry reductions are derived. Furthermore, the conservation laws of the equation are constructed.
\end{abstract}

Keywords: logarithmic-KP equation; Lie symmetry method; explicit solutions; conservation laws

\section{Introduction}

Nonlinear evolution equations (NLEEs) have been used in many science fields, such as physics, chemistry, engineering, and other fields. The investigation of the explicit solutions of NLEEs gave rise to much research work. A great many of systematic and effective methods are used for investigating NLEEs. Some of the methods are the inverse scattering method [1], the Hirota bilinear method [2, 3], the Bäklund transformation method [4-6], Darboux transformation [7, 8], the Painlevé analysis [9], the Lie group method [10-28], the solitary wave ansatz method [29-36], and others.

Conservation laws (CLs) provide a important tool to investigate many problems involving mathematical physics. A systematic method for the determination of conservation laws is the famous Noether theorem [37]. Recently, the direct method was given in [11], a new method to construct the conservation laws was provided in [38].

The generalized KP equation is the so-called logarithmic-KP $(\log -\mathrm{KP})$ equation given by [39]

$$
\left(v_{t}+(v \ln |v|)_{x}+v_{x x x}\right)_{x}+v_{y y}=0
$$

where $v(x, t)$ represents the wave profile. In [39], the authors studied the Gaussian solitary waves of the log-KP equation. More explications of the log-KP equation and its applications can be found in [39] and references therein. In [25], the authors studied the log-KdV equation. The KP equation appears in many important fields, such as water waves, ferromagnetic media, and so on. Kadomtsev and Petviashvili first derived the famous KP equation [40]. There are many papers dealing with these types of equations [31-36, 39, 40]. We first employ the following transformation [39]:

$$
v=e^{u},
$$

(c) 2016 Wang and Deng. This article is distributed under the terms of the Creative Commons Attribution 4.0 International License (http://creativecommons.org/licenses/by/4.0/), which permits unrestricted use, distribution, and reproduction in any medium, provided you give appropriate credit to the original author(s) and the source, provide a link to the Creative Commons license, and indicate if changes were made. 
we get

$$
\begin{aligned}
& u_{x t}+u_{x x}+u_{y y}+u_{x x x x}+3 u_{x x}^{2}+u_{y}^{2} \\
& \quad+3 u_{x} u_{x x x}+u_{x}^{2}+u u_{x x}+3 u_{x}^{2} u_{x x}=0
\end{aligned}
$$

In this paper, we use the Lie group method to deal with (3). The outline of the paper is as follows: In Section 2, the vectors fields are derived. In Section 3, symmetry reductions and explicit solutions are constructed. In Section 4, conservation laws are presented using the new conservation law theorem. The conclusions are presented in the final section.

\section{Lie symmetry analysis}

Suppose that (3) is invariant via the one-parameter Lie group

$$
\begin{aligned}
& t^{*}=t+\varepsilon \xi_{t}(x, y, t, u)+O\left(\varepsilon^{2}\right), \\
& x^{*}=x+\varepsilon \xi_{x}(x, y, t, u)+O\left(\varepsilon^{2}\right), \\
& y^{*}=y+\varepsilon \xi_{y}(x, y, t, u)+O\left(\varepsilon^{2}\right), \\
& u^{*}=u+\varepsilon \eta(x, y, t, u)+O\left(\varepsilon^{2}\right),
\end{aligned}
$$

where $\varepsilon$ is the group parameter, and the vector fields are

$$
V=\xi_{t}(x, y, t, u) \frac{\partial}{\partial t}+\xi_{x}(x, y, t, u) \frac{\partial}{\partial x}+\xi_{y}(x, y, t, u) \frac{\partial}{\partial y}+\eta(x, y, t, u) \frac{\partial}{\partial u} .
$$

Here

$$
\begin{array}{ll}
\xi_{t}(x, y, t, u)=\left.\frac{d t^{*}}{d \varepsilon}\right|_{\varepsilon=0}, & \xi_{x}(x, y, t, u)=\left.\frac{d x^{*}}{d \varepsilon}\right|_{\varepsilon=0}, \\
\xi_{y}(x, y, t, u)=\left.\frac{d y^{*}}{d \varepsilon}\right|_{\varepsilon=0}, & \eta(x, y, t, u)=\left.\frac{d u^{*}}{d \varepsilon}\right|_{\varepsilon=0} .
\end{array}
$$

Under the assumption of the infinitesimal invariance criterion, one gets

$$
\left.p r^{(3)} V(\Delta)\right|_{\Delta=0}=0
$$

According to the Lie group theory, one has

$$
\begin{aligned}
& \eta^{x t}+\eta^{x x}+\eta^{y y}+\eta^{x x x x}+6 \eta^{x x} u_{x x}+2 u_{y} \eta^{y}+3 \eta^{x} u_{x x x} \\
& \quad+3 u_{x} \eta^{x x x}+2 u_{x} \eta^{x}+\eta u_{x x}+u \eta^{x x}+6 u_{x} u_{x x} \eta^{x}+3 u_{x}^{2} \eta^{x x}=0
\end{aligned}
$$

Putting (4) into (8), and letting all of the powers of derivatives of $u$ be zero, one can obtain overdetermined systems. Solving the systems, one can get

$$
\xi_{t}=c_{1}, \quad \xi_{x}=c_{3} F(t), \quad \xi_{y}=c_{2}, \quad \eta=c_{3} F_{t},
$$


where $c_{1}, c_{2}$, and $c_{3}$ are arbitrary constants, and $F$ is a smooth function of $t$. Consequently, we have

$$
V_{1}=\frac{\partial}{\partial t}, \quad V_{2}=\frac{\partial}{\partial y}, \quad V_{3}=F \frac{\partial}{\partial x}+F_{t} \frac{\partial}{\partial u} .
$$

In addition, solving the Lie equation

$$
\begin{array}{lll}
\frac{d(\bar{x}(\varepsilon))}{d \varepsilon}=\xi_{x}(\bar{x}(\varepsilon), \bar{y}(\varepsilon), \bar{t}(\varepsilon), \bar{u}(\varepsilon)), & \bar{x}(0)=x, \\
\frac{d(\bar{y}(\varepsilon))}{d \varepsilon}=\xi_{y}(\bar{x}(\varepsilon), \bar{y}(\varepsilon), \bar{t}(\varepsilon), \bar{u}(\varepsilon)), & \bar{y}(0)=y, \\
\frac{d(\bar{t}(\varepsilon))}{d \varepsilon}=\xi_{t}(\bar{x}(\varepsilon), \bar{y}(\varepsilon), \bar{t}(\varepsilon), \bar{u}(\varepsilon)), & \bar{t}(0)=t, \\
\frac{d(\bar{u}(\varepsilon))}{d \varepsilon}=\eta(\bar{x}(\varepsilon), \bar{y}(\varepsilon), \bar{t}(\varepsilon), \bar{u}(\varepsilon)), & \bar{u}(0)=u,
\end{array}
$$

where $\varepsilon$ is a group parameter, we get the Lie symmetry group,

$$
g:(x, y, t, u) \rightarrow(\bar{x}, \bar{y}, \bar{t}, \bar{u}) .
$$

The associated one-parameter groups $g_{i}(\varepsilon)$ generated by $V_{i}$ for $i=1,2,3$ are

$$
\begin{aligned}
& g_{1}:(x, y, t, u) \mapsto(x, t+\varepsilon, y, u), \\
& g_{2}:(x, y, t, u) \mapsto(x, t, y+\varepsilon, y, u), \\
& g_{3}:(x, y, t, u) \mapsto\left(x+F \varepsilon, y, t, u+F_{t} \varepsilon\right) .
\end{aligned}
$$

In addition, we get the following associated theorem.

Theorem 1 If $u=f(x, y, t)$ is a solution of the logarithmic-KP equation, the functions

$$
\begin{aligned}
& g_{1}(\varepsilon) \cdot f(x, y, t)=f(x, y, t-\varepsilon), \\
& g_{2}(\varepsilon) \cdot f(x, y, t)=f(x, y-\varepsilon, t), \\
& g_{3}(\varepsilon) \cdot f(x, y, t)=f(x-F \varepsilon, y, t)+F_{t} \varepsilon,
\end{aligned}
$$

are also solutions of (3).

Taking the following Gaussian solitary wave solution [30]:

$$
u(x, t)=\frac{c}{k}+\frac{1}{2}-\frac{3 k^{2}+2 r^{2}}{12 k^{4}}(k x+r y-c t)^{2},
$$

we can derive a new explicit solution of (3) using $g_{3}$,

$$
u(x, t)=\frac{c}{k}+\frac{1}{2}-\frac{3 k^{2}+2 r^{2}}{12 k^{4}}(k(x-F \varepsilon)+r y-c t)^{2}+F_{t} \varepsilon .
$$

Hence, one can get new solutions of (1),

$$
v(x, t)=e^{\frac{c}{k}+\frac{1}{2}-\frac{1}{4 k^{2}}(k(x-F \varepsilon)+r y-c t)^{2}+F_{t} \varepsilon} .
$$


In particular, letting $F(t)=t^{2}$, one can get

$$
\nu(x, t)=e^{\frac{c}{k}+\frac{1}{2}-\frac{1}{4 k^{2}}\left(k\left(x-t^{2} \varepsilon\right)+r y-c t\right)^{2}+2 t \varepsilon},
$$

setting $F(t)=\sin t$, one has

$$
v(x, t)=e^{\frac{c}{k}+\frac{1}{2}-\frac{1}{4 k^{2}}(k(x-\sin t \varepsilon)+r y-c t)^{2}+\cos t \varepsilon},
$$

and setting $F(t)=\tanh t$, one obtains

$$
v(x, t)=e^{\frac{c}{k}+\frac{1}{2}-\frac{1}{4 k^{2}}(k(x-\tanh t \varepsilon)+r y-c t)^{2}+\left(1-\tanh t^{2}\right) \varepsilon},
$$

setting $F(t)=e^{t}$, one can arrive at

$$
v(x, t)=e^{\frac{c}{k}+\frac{1}{2}-\frac{1}{4 k^{2}}\left(k\left(x-e^{t} \varepsilon\right)+r y-c t\right)^{2}+e^{t} \varepsilon},
$$

setting $F(t)=\sin \left(e^{t}\right)$, one can lead to

$$
v(x, t)=e^{\frac{c}{k}+\frac{1}{2}-\frac{1}{4 k^{2}}\left(k\left(x-\sin \left(e^{t}\right) \varepsilon\right)+r y-c t\right)^{2}+\cos \left(e^{t}\right) e^{t} \varepsilon},
$$

setting $F(t)=\ln (t)$, one can have

$$
v(x, t)=e^{\frac{c}{k}+\frac{1}{2}-\frac{1}{4 k^{2}}(k(x-\ln (t) \varepsilon)+r y-c t)^{2}+\frac{1}{t} \varepsilon} .
$$

Remark 1 Many new explicit solutions can be derived via the solutions obtained [30].

\section{Symmetry reductions and explicit solutions}

\subsection{Symmetry reductions}

In the present subsection, we will present symmetry reductions and explicit solutions of (3).

(1) $V_{1}$.

For the generator $V_{1}$, we have

$$
f_{x x}+f_{y y}+f_{x x x x}+3 f_{x x}^{2}+f_{y}^{2}+3 f_{x} f_{x x x}+f_{x}^{2}+\not f_{x x}+3 f_{x}^{2} f_{x x}=0 .
$$

For this equation, we found that it also is a PDE. In order to reduce this equation, once again, we use the Lie group method to deal with this equation. As in the previous step, one can get the corresponding vectors,

$$
\Upsilon_{1}=\frac{\partial}{\partial x}, \quad \Upsilon_{2}=\frac{\partial}{\partial y} .
$$

(1.1) $\Upsilon_{1}$

For $\Upsilon_{1}$, we have

$$
g_{y y}+g_{y}^{2}=0 .
$$


Solving this equation, one can get

$$
g=\ln \left(c_{1} y+c_{2}\right)
$$

That is to say,

$$
u=\ln \left(c_{1} y+c_{2}\right)
$$

Also, one can get

$$
v=c_{1} y+c_{2} \text {. }
$$

(1.2) $\Upsilon_{2}$

For $\Upsilon_{2}$, we get

$$
g_{x x}+g_{x x x x}+3 g_{x x}^{2}+3 g_{x} g_{x x x}+g_{x}^{2}+g g_{x x}+3 g_{x}^{2} g_{x x}=0 .
$$

(2) $V_{2}$.

In the case of $V_{2}$, we get the group-invariant solution,

$$
\begin{aligned}
& u=f(x, t), \\
& f_{x t}+f_{x x}+f_{x x x x}+3 f_{x x}^{2}+3 f_{x} f_{x x x}+f_{x}^{2}+f f_{x x}+3 f_{x}^{2} f_{x x}=0 .
\end{aligned}
$$

As in the previous step, we get the associated vectors,

$$
\Gamma_{1}=\frac{\partial}{\partial x}, \quad \Gamma_{2}=\frac{\partial}{\partial t} .
$$

(2.1) $\Gamma_{1}$

For $\Gamma_{1}$, we get the trivial solution,

$$
u=c_{1} \text {. }
$$

(2.2) $\Gamma_{2}$.

For $\Gamma_{2}$, we arrive at

$$
g_{x x}+g_{x x x x}+3 g_{x x}^{2}+3 g_{x} g_{x x x}+g_{x}^{2}+g g_{x x}+3 g_{x}^{2} g_{x x}=0 .
$$

(3) $V_{3}$.

For this case, we get

$$
u=\frac{g(y, t)}{F}+\frac{F_{t} x}{F}
$$

Plugging (36) into (3), one arrives at

$$
\frac{1}{F^{2}}\left(F F_{t t}+F g_{y y}+g_{y}^{2}\right)=0 .
$$


By solving this equation, one obtains

$$
g=-\frac{1}{2} \ln \left(\frac{F_{t t} F}{\left(F_{1} \sin \left(\frac{\sqrt{F_{t t}} y}{\sqrt{F}}\right)-F_{2} \cos \left(\frac{\sqrt{F_{t t}} y}{\sqrt{F}}\right)\right)^{2}}\right) F .
$$

In this way, we get

$$
u=-\frac{1}{2} \ln \left(\frac{F_{t t} F}{\left(F_{1} \sin \left(\frac{\sqrt{F_{t t}} y}{\sqrt{F}}\right)-F_{2} \cos \left(\frac{\sqrt{F_{t t}} y}{\sqrt{F}}\right)\right)^{2}}\right)+\frac{F_{t} x}{F} .
$$

Thus, one can get the explicit solution of (1),

$$
\begin{aligned}
v & =e^{u}=e^{-\frac{1}{2} \ln \left(\frac{F_{t t} F}{\left(F_{1} \sin \left(\frac{\sqrt{F_{t t}} y}{\sqrt{F}}\right)-F_{2} \cos \left(\frac{\sqrt{F_{t t}} y}{\sqrt{F}}\right)\right)^{2}}\right)+\frac{F_{t} x}{F}} \\
& =\left(\frac{F_{t t} F}{\left(F_{1} \sin \left(\frac{\sqrt{F_{t t}} y}{\sqrt{F}}\right)-F_{2} \cos \left(\frac{\sqrt{F_{t t}} y}{\sqrt{F}}\right)\right)^{2}}\right)^{-\frac{1}{2}} e^{\frac{F_{t} x}{F}}
\end{aligned}
$$

where $F_{t t} \neq 0, F_{1}$ and $F_{2}$ are functions of $t$.

In particular, if we set $F=t$, we have

$$
u=\frac{g(y, t)}{t}+\frac{x}{t}
$$

Substituting (41) into (3), we obtain

$$
\frac{1}{t^{2}}\left(t g_{y y}+g_{y}^{2}\right)=0
$$

Solving this equation, one can get

$$
g=t \ln \left(\frac{F_{1}(t) y+F_{2}(t)}{t}\right) \text {. }
$$

Therefore, one gets

$$
u=\ln \left(\frac{F_{1}(t) y+F_{2}(t)}{t}\right)+\frac{x}{t}
$$

Therefore, one can derive the explicit solution of (1),

$$
v=e^{u}=\left(\frac{F_{1}(t) y+F_{2}(t)}{t}\right) e^{\frac{x}{t}}
$$

(3) $V_{1}+V_{2}$.

For this case, we get

$$
u=f(\xi, \tau), \quad \xi=x, \quad \tau=y-t .
$$

Plugging (46) into (3), one arrives at

$$
-f_{\xi \tau}+f_{\xi \xi}+f_{\tau \tau}+f_{\xi \xi \xi \xi}+3 f_{\xi \xi}^{2}+f_{\tau}^{2}+3 f_{\xi} f_{\xi \xi \xi}+f_{\xi}^{2}+\not f \xi \xi+3 f_{\xi}^{2} f_{\xi \xi}=0 .
$$


As in the previous step, we obtain the associated vectors,

$$
\Gamma_{1}=\frac{\partial}{\partial \xi}, \quad \Gamma_{2}=\frac{\partial}{\partial \tau} .
$$

(3.1) $\Gamma_{1}$.

For $\Gamma_{1}$, we get

$$
f=g(\tau)
$$

and

$$
g_{\tau}^{2}+g_{\tau \tau}=0
$$

(3.2) $\Gamma_{2}$.

For $\Gamma_{2}$, we have

$$
g_{\xi \xi}+g_{\xi \xi \xi \xi}+3 g_{\xi \xi}^{2}+3 g_{\xi} g_{\xi \xi \xi}+g_{\xi}^{2}+g g_{\xi \xi}+3 g_{\xi}^{2} g_{\xi \xi}=0 .
$$

(3.3) $\Gamma_{2}+\lambda \Gamma_{1}$ (traveling wave transformation).

For this case, we get

$$
f=g(\pi), \quad \pi=\xi-\tau=x-\lambda(y-t)
$$

and

$$
\begin{aligned}
& \lambda g_{\pi \pi}+g_{\pi \pi}+\lambda^{2} g_{\pi \pi}+g_{\pi \pi \pi \pi}+3 g_{\pi \pi}^{2}+\lambda^{2} g_{\pi}^{2}+3 g_{\pi} g_{\pi \pi \pi} \\
& \quad+g_{\pi}^{2}+g g_{\pi \pi}+3 g_{\pi}^{2} g_{\pi \pi}=0 .
\end{aligned}
$$

\section{Conservation laws}

In the present section, we derive the conservation laws of the logarithmic-KP equation.

\subsection{Necessary preliminaries}

For a conserved vector the following conservation equation holds:

$$
D_{t}\left(C^{t}\right)+D_{x}\left(C^{x}\right)+D_{y}\left(C^{y}\right)=0
$$

where $C^{t}=C^{t}(t, x, y, u, \ldots), C^{x}=C^{x}(t, x, y, u, \ldots), C^{y}=C^{y}(t, x, y, u, \ldots)$.

A formal Lagrangian for (3) is

$$
\begin{aligned}
L= & p(x, y, t)\left[u_{x t}+u_{x x}+u_{y y}+u_{x x x x}+3 u_{x x}^{2}\right. \\
& \left.+u_{y}^{2}+3 u_{x} u_{x x x}+u_{x}^{2}+u u_{x x}+3 u_{x}^{2} u_{x x}\right] .
\end{aligned}
$$

Here $p(x, y, t)$ is a new dependent variable. 
Theorem 2 [29] Every Lie point, Lie-Bäcklund, and nonlocal symmetry of equation (3) provides a conservation law for this equation and the adjoint equation. Then the elements of conservation vector are given by the following formula:

$$
\begin{aligned}
C^{i}= & \xi^{i} L+W^{\alpha}\left[\frac{\partial L}{\partial u_{i}^{\alpha}}-D_{j}\left(\frac{\partial L}{\partial u_{i j}^{\alpha}}\right)+D_{j} D_{k}\left(\frac{\partial L}{\partial u_{i j k}^{\alpha}}\right)-\cdots\right]+D_{j}\left(W^{\alpha}\right)\left[\left(\frac{\partial L}{\partial u_{i j}^{\alpha}}\right)\right. \\
& \left.-D_{k}\left(\left(\frac{\partial L}{\partial u_{i j k}^{\alpha}}\right)\right)+\cdots\right]+D_{j} D_{k}\left(W^{\alpha}\right)\left[\frac{\partial L}{\partial u_{i j k}^{\alpha}}-\cdots\right],
\end{aligned}
$$

where $W^{\alpha}=\eta^{\alpha}-\xi^{j} u_{j}^{\alpha}$.

\subsection{Conservation laws}

The adjoint equation of (3) has the form

$$
\begin{aligned}
F= & -2 u_{y} p_{y}-2 p u_{y y}+p_{y y}+p_{x t}+6 u_{x} p_{x} u_{x x}+p_{x x}+u p_{x x} \\
& +3 u_{x}^{2} p_{x x}-3 u_{x x} p_{x x}-3 u_{x} p_{x x x}+p_{x x x x}=0 .
\end{aligned}
$$

It is easily found that on substituting $u$ instead of $p$ in equation (57), equation (3) is not recovered. Thus, equation (3) is not self-adjoint. The Lagrangian is

$$
\begin{aligned}
L= & p\left[u_{x t}+u_{x x}+u_{y y}+u_{x x x x}+3 u_{x x}^{2}+u_{y}^{2}\right. \\
& \left.+3 u_{x} u_{x x x}+u_{x}^{2}+u u_{x x}+3 u_{x}^{2} u_{x x}\right] .
\end{aligned}
$$

From (56), one gets

$$
\begin{aligned}
C^{t}= & \xi_{t} L+W\left(-D_{x} \frac{\partial L}{\partial u_{t x}}\right)+D_{x}(W) \frac{\partial L}{\partial u_{t x}} \\
C^{x}= & \xi L+W\left(\frac{\partial L}{\partial u_{x}}-D_{x} \frac{\partial L}{\partial u_{x x}}-D_{t} \frac{\partial L}{\partial u_{x t}}+D_{x}^{2} \frac{\partial L}{\partial u_{x x x}}-D_{x}^{3}(W) \frac{\partial L}{\partial u_{x x x x}}\right) \\
& +D_{x}(W)\left(\frac{\partial L}{\partial u_{x x}}-D_{x} \frac{\partial L}{\partial u_{x x x}}+D_{x}^{2} \frac{\partial L}{\partial u_{x x x x}}\right) \\
& +D_{x}^{2}(W)\left(\frac{\partial L}{\partial u_{x x x}}-D_{x}(W) \frac{\partial L}{\partial u_{x x x x}}\right) \\
C^{y}= & \xi_{y} L+W\left(\frac{\partial L}{\partial u_{y}}-D_{y} \frac{\partial L}{\partial u_{y y}}\right)+D_{y}(W) \frac{\partial L}{\partial u_{y y}}
\end{aligned}
$$

which leads to

$$
\begin{aligned}
C^{t}= & -v_{x} W+v W_{x}, \\
C^{x}= & W\left(u_{x} v-v_{x}-v_{x} u-3 u_{x}^{2} v_{x}-v_{t}-v_{x x x}+3 u_{x} v_{x x}\right) \\
& +\left(W_{x}\right)\left(v+3 v u_{x x}+u v+3 u_{x}^{2} v-3 u_{x} v_{x}+v_{x x}\right) \\
& +W_{x x}\left(3 u_{x} v-v_{x}\right), \\
C^{y}= & 2 v W u_{y}-W v_{y}+v W_{y} .
\end{aligned}
$$


In particular:

1. For the case $V_{1}=\partial_{y}$, we get $W=-u_{y}$, and

$$
\begin{aligned}
C^{t}= & v_{x} u_{y}-v u_{y x} \\
C^{x}= & -u_{y}\left(u_{x} v-v_{x}-v_{x} u-3 u_{x}^{2} v_{x}-v_{t}-v_{x x x}+3 u_{x} v_{x x}\right) \\
& -u_{x y}\left(v+3 v u_{x x}+u v+3 u_{x}^{2} v-3 u_{x} v_{x}+v_{x x}\right) \\
& -u_{y x x}\left(3 u_{x} v-v_{x}\right) \\
C^{y}= & -2 v u_{y}^{2}+u_{y} v_{y}-v u_{y y} .
\end{aligned}
$$

2. For $V_{2}=\partial_{t}$, one has $W=-u_{t}$, and

$$
\begin{aligned}
C^{t}= & u_{t} v_{x}-v u_{t x}, \\
C^{x}= & -u_{t}\left(u_{x} v-v_{x}-v_{x} u-3 u_{x}^{2} v_{x}-v_{t}-v_{x x x}+3 u_{x} v_{x x}\right) \\
& -\left(u_{t x}\right)\left(v+3 v u_{x x}+u v+3 u_{x}^{2} v-3 u_{x} v_{x}+v_{x x}\right) \\
& -u_{t x x}\left(3 u_{x} v-v_{x}\right), \\
C^{y}= & -2 v u_{t} u_{y}+u_{t} v_{y}-v u_{t y} .
\end{aligned}
$$

3. For the case $V_{3}=F \partial_{x}+F_{t} \partial_{u}$, we have $W=F_{t}-F u_{x}$, and we arrive at

$$
\begin{aligned}
C^{t}= & -v_{x}\left(F_{t}-F u_{x}\right)+v\left(F_{t}-F u_{x}\right)_{x} \\
= & F v_{x} u_{x}-F_{t} v_{x}+F_{t x} v-F_{x} v u_{x}-F v u_{x x}, \\
C^{x}= & \left(F_{t}-F u_{x}\right)\left(u_{x} v-v_{x}-v_{x} u-3 u_{x}^{2} v_{x}-v_{t}-v_{x x x}+3 u_{x} v_{x x}\right) \\
& +\left(F_{t x}-F_{x} u_{x}-F u_{x t}\right)\left(v+3 v u_{x x}+u v+3 u_{x}^{2} v-3 u_{x} v_{x}+v_{x x}\right) \\
& +\left(F_{t x x}-F_{x x} u_{x}-F_{x} u_{x x}-F_{x} u_{x t}-F u_{x x t}\right)\left(3 u_{x} v-v_{x}\right), \\
C^{y}= & 2 v W u_{y}-W v_{y}+v W_{y}, \\
= & 2 v u_{y} F_{t}-2 v u_{y} F u_{x}-F_{t} v_{y}+F u_{x} v_{y}+v F_{t y}-v F_{y} u_{x}-F v u_{x y} .
\end{aligned}
$$

\section{Concluding remarks}

In this paper, we studied the logarithmic-KP equation. The Lie group method was applied to conduct the analysis for this work. Symmetry reductions and explicit solutions were obtained. These solutions maybe explain some complex physical phenomena. It is to be noted that conservation laws were also constructed. We hope that the results obtained may be useful in further numerical analysis. Comparing with [30], it can be seen that our results are new. In the future work, we will try to employ more methods, such as nonclassical Lie groups, the nonlocal symmetry method, and other methods, to derive more novel exact solutions of the logarithmic types of equations. 


\section{Acknowledgements}

The authors would like to thank to the editor and reviewers for their constructive comments and suggestions that improved this paper. This paper is funded by International Graduate Exchange Program of Beijing Institute of Technology.

Received: 29 March 2016 Accepted: 23 August 2016 Published online: 01 September 2016

\section{References}

1. Ablowitz, MJ, Segur, H: Solitons and Inverse Scattering Transform. SIAM, Philadelphia (1981)

2. Hirota, R: The Direct Method in Soliton Theory. Cambridge University Press, Cambridge (2004)

3. LV, X, Peng, MS: Nonautonomous motion study on accelerated and decelerated solitons for the variable-coefficient Lenells-Fokas model. Chaos 23, 013122 (2013)

4. Ma, WX, et al.: Bäcklund transformation and its superposition principle of a Blaszak-Marciniak four-field lattice. J. Math. Phys. 40, 6071-6086 (1999)

5. Tian, B, Gao, YT, Zhu, HW: Variable-coefficient higher-order nonlinear Schrodinger model in optical fibers: variable-coefficient bilinear form, Backlund transformation, brightons and symbolic computation. Phys. Lett. A 366 223-229 (2007)

6. Gao, YT, Tian, B: On the non-planar dust-ion-acoustic waves in cosmic dusty plasmas with transverse perturbations Europhys. Lett. 77, 15001 (2007)

7. Li, YS, Ma, WX, Zhang, JE: Darboux transformations of classical Boussinesq system and its new solutions. Phys. Lett. A $275,60-66(2000)$

8. LV, X: Soliton behavior for a generalized mixed nonlinear Schrodinger model with $\mathrm{N}$-fold Darboux transformation. Chaos 23, 033137 (2013)

9. Lou, SY, Wu, QX: Painlevé integrability of two sets of nonlinear evolution equations with nonlinear dispersions. Phys. Lett. A 262, 344-349 (1999)

10. Ibragimov, NH (ed.): CRC Handbook of Lie Group Analysis of Differential Equations, Vols. 1-3. CRC Press, Boca Raton (1994)

11. Bluman, GW, Cheviakov, A, Anco, S: Applications of Symmetry Methods to Partial Differential Equations. Springer, New York (2010)

12. Olver, PJ: Application of Lie Group to Differential Equation. Springer, New York (1986)

13. Ovsiannikov, LV: Group Analysis of Differential Equations. Academic Press, New York (1982)

14. Wang, GW, Xu, TZ, Ebadi, G, Johnson, S, Strong, AJ, Biswas, A: Singular solitons, shock waves, and other solutions to potential KdV equation. Nonlinear Dyn. 76, 1059-1068 (2014)

15. Wang, GW, Xu, TZ, Johnson, S, Biswas, A: Solitons and Lie group analysis to an extended quantum Zakharov-Kuznetsov equation. Astrophys. Space Sci. 349, 317-327 (2014)

16. Wang, GW, Liu, XQ, Zhang, YY: Symmetry reduction, exact solutions and conservation laws of a new fifth-order nonlinear integrable equation. Commun. Nonlinear Sci. Numer. Simul. 18, 2313-2320 (2013)

17. Vaneeva, OO, Kuriksha, O, Sophocleous, C: Enhanced group classification of Gardner equations with time-dependent coefficients. Commun. Nonlinear Sci. Numer. Simul. 22, 1243-1251 (2015)

18. Wang, GW, Xu, TZ, Abazari, R, Jovanoski, Z, Biswas, A: Shock waves and other solutions to the Benjamin-Bona-Mahoney-Burgers equation with dual power-law nonlinearity. Acta Phys. Pol. A 126, 1221-1225 (2014)

19. Wang, GW, Xu, TZ, Liu, XQ: New explicit solutions of the fifth-order KdV equation with variable coefficients. Bull. Malays. Math. Soc. 37, 769-778 (2014)

20. Wang, GW, Liu, XQ, Zhang, YY: Lie symmetry analysis to the time fractional generalized fifth-order KdV equation. Commun. Nonlinear Sci. Numer. Simul. 18, 2321-2326 (2013)

21. Wang, GW, Kara, AH: Conservation laws, multipliers, adjoint equations and Lagrangians for Jaulent-Miodek and some families of systems of KdV type equations. Nonlinear Dyn. 81, 753-763 (2015)

22. Wang, GW, Kara, AH: Nonlocal symmetry analysis, explicit solutions and conservation laws for the fourth-order Burgers' equation. Chaos Solitons Fractals 81, 290-298 (2015)

23. Wang, GW, Kara, AH, Fakhar, K: Symmetry analysis and conservation laws for the class of time fractional nonlinear dispersive equation. Nonlinear Dyn. 82, 281-287 (2015)

24. Wang, GW, Fakhar, K: Lie symmetry analysis, nonlinear self-adjointness and conservation laws to an extended $(2+1)$-dimensional Zakharov-Kuznetsov-Burgers equation. Comput. Fluids 119, 143-148 (2015)

25. Wang, GW, Xu, TZ: Group analysis, explicit solutions and conservation laws of the logarithmic-KdV equation. J. Korean Phys. Soc. 66, 1475-1481 (2015)

26. Wang, GW, Xu, TZ, Biswas, A: Topological solitons and conservation laws of the coupled Burgers equation. Rom. Rep. Phys. 66, 274-285 (2014)

27. Wang, GW, Kara, AH, Buhe, E, Fakhar, K: Group analysis and conservation laws of coupled system for the carbon nanotubes conveying fluid. Rom. J. Phys. 60, 952-960 (2015)

28. Wang, GW, Kara, AH, Fakhar, K, Vega-Guzman, J, Biswas, A: Group analysis, exact solutions and conservation laws of a generalized fifth order KdV equation. Chaos Solitons Fractals 86, 8-15 (2016)

29. Fabian, AL, Kohl, R, Biswas, A: Perturbation of topological solitons due to sine-Gordon equation and its type. Commun. Nonlinear Sci. Numer. Simul. 14, 1227-1244 (2009)

30. Collins, T, Kara, AH, Bhrawy, AH, Triki, H, Biswas, A: Dynamics of shallow water waves with logarithmic nonlinearity. Rom. Rep. Phys. 68(3) (2016, in press)

31. Biswas, A, Ranasinghe, A: 1-Soliton solution of Kadomtsev-Petviashvili equation with power law nonlinearity. Appl. Math. Comput. 214, 645-647 (2009)

32. Jawad, AJM, Petkovic, M, Biswas, A: Soliton solutions for nonlinear Calaogero-Degasperis and potential Kadomtsev-Petviashvili equations. Comput. Math. Appl. 62, 2621-2628 (2011)

33. Triki, H, Sturdevant, BJM, Hayat, T, Aldossary, OM, Biswas, A: Shock wave solutions of the variants of the Kadomtsev-Petviashvili equation. Can. J. Phys. 89, 979-984 (2011)

34. Bhrawy, AH, Abdelkawy, MA, Kumar, S, Biswas, A: Solitons and other solutions to Kadomtsev-Petviashvili equation of B-type. Rom. J. Phys. 58, 729-748 (2013) 
35. Ebadi, G, Fard, NY, Bhrawy, AH, Kumar, S, Triki, H, Yildirim, A, Biswas, A: Solitons and other solutions to the $(3+1)$-dimensional extended Kadomtsev-Petviashvili equation with power law nonlinearity. Rom. Rep. Phys. 65, 27-62 (2013)

36. Fard, NY, Foroutan, MR, Eslami, M, Mirzazadeh, M, Biswas, A: Solitary waves and other solutions to Kadomtsev-Petviashvili equation with spatio-temporal dispersion. Rom. J. Phys. 60, 1337-1360 (2015)

37. Noether, E, Variationsprobleme, I: Nachr. Akad. Wiss. Gött. Math.-Phys. KI. 2, 235-257 (1918); English translation in Transp. Theory Stat. Phys. 1, 186-207 (1971)

38. Ibragimov, NH: A new conservation theorem. J. Math. Anal. Appl. 333, 311-328 (2007)

39. Wazwaz, AM: Gaussian solitary waves for the logarithmic-KdV and the logarithmic-KP equations. Phys. Scr. 89, 095206 (2014)

40. Kadomtsev, BB, Petviashvili, VI: On the stability of solitary waves in weakly dispersive media. Sov. Phys. Dokl. 15 539-541 (1970)

Submit your manuscript to a SpringerOpen ${ }^{\circ}$ journal and benefit from:

- Convenient online submission

Rigorous peer review

- Immediate publication on acceptance

- Open access: articles freely available online

- High visibility within the field

- Retaining the copyright to your article 